\title{
Pascale-Marie MILAN, Tourisme et changement social chez les Na de Chine. Étude comparée d'une coutume sexuelle: le séssé
}

Thèse de doctorat en anthropologie, Université Lyon 2, Université Laval, sous la direction de Lionel obadia et Jean Michaud, soutenue le 17 septembre 2019

Tourism and social change among the Na of China. A comparative study of a sexual custom: the séssé

\section{Pascale-Marie Milan}

\section{CpenEdition}

Édition électronique

URL : http://journals.openedition.org/tourisme/3416

DOI : 10.4000/tourisme.3416

ISSN : 2492-7503

Éditeur

Éditions touristiques européennes

Référence électronique

Pascale-Marie Milan, « Pascale-Marie mILAN, Tourisme et changement social chez les Na de Chine. Étude comparée d'une coutume sexuelle : le séssé ", Mondes du Tourisme [En ligne], 18|2020, mis en ligne le 01 mars 2021, consulté le 15 mars 2021. URL : http://journals.openedition.org/tourisme/3416 ; DOI https://doi.org/10.4000/tourisme.3416

Ce document a été généré automatiquement le 15 mars 2021.

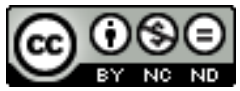

Mondes du tourisme est mis à disposition selon les termes de la licence Creative Commons Attribution - Pas d'Utilisation Commerciale - Pas de Modification 4.0 International. 


\section{Pascale-Marie MILAN, Tourisme et} changement social chez les Na de Chine. Étude comparée d'une coutume sexuelle : le séssé

Thèse de doctorat en anthropologie, Université Lyon 2, Université Laval, sous la direction de Lionel Obadia et Jean Michaud, soutenue le 17 septembre 2019

Tourism and social change among the Na of China. A comparative study of a sexual custom: the séssé

Pascale-Marie Milan

Depuis qu'a débuté la politique d'ouverture économique de la Chine (gaige kaifang - 改 革开放1), le tourisme est devenu un outil stratégique du gouvernement chinois pour 《 développer » les shaoshu minzu (少数民族 - minorités nationales). Alors que nombre de nationalités minoritaires des marges sino-tibétaines organisaient leurs moyens de subsistance autour de l'agriculture et de l'élevage, le développement du tourisme les a faites entrer dans une économie de marché, à la marge de laquelle ils demeuraient auparavant. Les $\mathrm{Na}$ de Chine, bien connus en anthropologie pour être un cas exemplaire des sociétés matrilinéaires et matrilocales, plus régulièrement présentés comme une société sans père ni mari (Cai, 1997), ne font pas exception. Comme bien d'autres minorités nationales, les $\mathrm{Na}$, plus communément appelés Mosuo en mandarin, sont considérés «en manque » de civilisation (Harrell, 1995). Participer au développement touristique est pour eux un moyen d'accroître leur niveau de vie, leur permettant de correspondre autant que possible aux nouveaux idéaux de modernité. Depuis les années 1990, la région du lac Lugu, située à la frontière du Yunnan et du Sichuan, a fait l'objet d'une mise en tourisme spectaculaire. Chaque année, des milliers de touristes chinois $(95 \%)$ viennent pour un ou deux jours visiter ces paysages d'eau et de montagne (Shanshui) et rencontrer ce groupe minoritaire considéré comme 
《arriéré" (luohou- 落后) et "primitif» (yuanshi - 原始), en raison du discours idéologique évolutionniste et de l'étude culturelle ambiante relative aux minorités nationales. Leur coutume de visite nocturne (séssé), institution qui prévoit la visite des hommes chez les femmes la nuit, s'est très vite trouvée réifiée par le dispositif touristique comme un attribut central de leur identité culturelle.

2 À partir d'un terrain effectué dans deux villages na pendant onze mois et demi entre 2012 et 2014, cette thèse s'est attachée à documenter le changement social et notamment les transformations de la vie intime en proposant une analyse comparée de ces deux villages, l'un touristique, l'autre non. L'objectif a été d'interroger les dimensions affectives, économiques et sociales qui arriment les sociabilités sexuelles aux configurations des maisonnées pour comprendre la recomposition de ces dernières en situation touristique. L'ethnographie met en lumière la maisonnée comme une unité sociale de base de l'organisation sociale se référant à la fois à la parenté et à l'économie domestique. Les maisonnées sont généralement constituées de 5 à 20 membres d'ascendance matrilinéaire. Les discours tenus aux touristes sur la scène publique et les discours tenus au quotidien par les $\mathrm{Na}$ sur la scène privée rendaient cependant la situation d'enquête labile. Pour me défaire des grandes catégories auxquelles j'étais assignée (touriste, occidentale) et prendre un positionnement me permettant d'accéder au texte caché (Scott, 2008) de la scène privée, j'ai rendu compte dans le premier chapitre de la réflexivité déployée in situ. Sur la scène publique, les $\mathrm{Na}$ tenaient un discours conforme au métarécit chinois. En plus des projections exotiques et érotiques dépeignant leur sexualité comme légère, les $\mathrm{Na}$ sont considérés comme les représentants du premier stade de l'évolution de l'humanité en raison de leur organisation sociale, autrement dit un matriarcat. En privé, d'autres discours et d'autres pratiques venaient cependant infléchir ces récits et notamment l'idée qu'il s'agirait d'une société sans père ni mari qui ne connaîtrait pas le mariage.

3 Pour rendre compte du changement social, ma thèse a d'abord proposé, au second chapitre, des éléments de discussion permettant de lire les conceptions indigènes de la parenté. En m'appuyant sur la notion de société à maison (Lévi-Strauss, 1979 ; Lamaison ,1987; Carsten et Hugh-Jones, 1995 ; Gillepsie, 2000), proposée comme un angle de lecture de la société na (Hsu, 1998), j'ai cherché à rendre compte des relations entre proches (relatedness) (Carsten, 2000). Au terme d'une discussion de la littérature anthropologique, je mets en évidence que le terme awo peut être considéré comme une unité sociale de base de l'organisation sociale. À la différence de Shih, qui considère le zidu comme l'unité sociale organisationnelle $(1993,2010)$, je montre que le terme awo, dont la signification recoupe en partie la notion de zidu, est utilisé dans le langage courant (au contraire du terme zidu) pour se référer à l'ensemble des personnes qui vivent dans la maison, les " gens de la maison » (awo hing), et à la construction en bois principale (awo zhimi). Cet angle de lecture offre l'avantage de sortir d'une représentation commune selon laquelle les Na seraient une société sans père ni mari (Cai, 1997). Plutôt que de réduire la variété des configurations observables à des généralités qui n'informent en rien la complexité sociale, la thèse a montré l'existence de termes pour désigner les géniteurs (ave) ou les partenaires sexuels des femmes (hrantchouba) ainsi que des pratiques de captation de partenaires sexuels ou d'adoption. Ces stratégies, trop peu mises en lumière, sont liées aux configurations des maisonnées et rendent compte avec finesse de la flexibilité de la parenté na. Malgré un mode de filiation matrilinéaire et pour rééquilibrer une balance genrée ou numérique 
défavorable, une maison peut recruter un membre féminin extérieur à la lignée en captant une partenaire sexuelle (tchumi ché, littéralement: "partenaire sexuelle de l'homme » « prendre ») - à condition que la maison donneuse n'en souffre pas et puisse assurer sa propre continuité. À l'inverse, les pratiques de captation de partenaires sexuels masculins se nomment zoma ché (littéralement « beau-fils " « prendre »). Moins courantes, elles visent généralement à rééquilibrer une balance entre genres quand la proportion d'hommes et de femmes dans une maison est inadéquate pour effectuer les gros travaux. L'adoption temporaire ou définitive de jeunes enfants issus d'un même lignage peut également pallier un manque générationnel de main d'œuvre ou assurer la continuité de la maison.

4 Sur la base de ces éléments et d'une présentation, au chapitre trois, du contexte chinois et des fils conducteurs permettant l'analyse du changement social, j'ai choisi d'utiliser le terme de maisonnée pour désigner l'awo, afin de montrer qu'elle n'est pas seulement au cœur de l'organisation sociale, mais qu'elle est la matrice d'une forme d'organisation intégrale, c'est-à-dire sociale, politique et économique. Le quatrième chapitre propose des portraits anthropologiques de quelques maisonnées représentatifs de la diversité de leurs compositions et resitue les histoires de peuplement des deux villages à l'étude dans le contexte géographique et historique régional et local. S'ensuit au chapitre cinq une analyse de la situation touristique. Cette formule, reprise à l'anthropologie dynamique de Balandier ([1951] 2008), explique le phénomène touristique en tant que situation sociale, économique et politique. Elle est apparue heuristique pour dépasser une lecture en termes d'impact ou de résistance et rendre compte des recompositions à l'œuvre. La notion a le double avantage de se référer à la fois à la "situation ethnologique ", afin d'interroger les stratégies rhétoriques des informateurs (Doquet, 2007), et aux situations concrètes vécues dans un contexte national. Ce chapitre s'attache ainsi à analyser la circulation des imaginaires, des personnes, des capitaux, des signes, etc., mettant en rapport des normes, des systèmes de valeur et des représentations différenciées entre les acteurs du tourisme. Il fournit des éléments pour comprendre les intérêts, les expériences et les relations vécues et montre combien l'activité se fonde sur une relation d'enchantement (Réau et Poupeau, 2007; Cousin et Réau, 2009). Apparaissent en creux des relations de pouvoir et des inégalités sociales, économiques et politiques qui participent d'une hiérarchisation des $\mathrm{Na}$ en termes de capital humain. Sur fond d'idéaux d'accès à la modernité de part et d'autre, une différenciation ethnique s'est opérée. Les $\mathrm{Na}$ de l'arrière-pays sont en effet devenus une main-d'œuvre bon marché pour les Na labellisés Mosuo du lac Lugu.

5 Le sixième chapitre propose une comparaison des économies morales entre les deux villages à l'étude. En documentant les pratiques économiques de subsistance et les nouvelles pratiques économiques apparues avec le tourisme, ce chapitre vient préciser les changements de l'organisation intégrale en maisonnée. Le système d'entraide donnant à voir l'étendue des liens dans le lignage, entre voisins et avec les hrantchouba (partenaires sexuels), et le système d'échange expliquent ainsi la forte charge morale et éthique des relations que les maisonnées entretiennent entre elles par une réciprocité généralisée et montrent que l'awo est un groupe de parenté à part entière. Ces systèmes montrent également à quel point les modalités des sociabilités sexuelles s'arriment à la configuration des maisonnées, tout en montrant une circulation de biens et de personnes, éphémère ou durable. L'entraide montre ainsi l'étendue du système intégral en maisonnée et donne à voir les relations intimes en tant que modalité de la reproduction sociale et biologique, qu'il s'agisse de l'institution de visite, du mariage ou 
de la cohabitation. Afin de continuer à faire prospérer leurs maisonnées, les Na se sont, en outre, adaptés à l'économie de marché en remodulant les pratiques d'entraide et d'échange qui participaient de la socialité na. L'activité touristique a cependant occasionné une mise en concurrence des maisons, au point que les formes de solidarité et d'obligation entre maisonnées parentes et amies ne maintiennent plus, ni ne structurent ou ne reproduisent l'organisation intégrale en maisonnée.

6 Le septième chapitre compare les festivités dans les deux villages. Celles-ci, en tant qu'institution politique régulatrice, révèlent la capacité du social à se créer et à s'instituer, voire à se réinventer dans le cas de la mise en tourisme. Ce sont soit des fêtes communautaires participant du lien social, soit des mises en scène à destination des touristes. En les considérant comme des arènes de jeux sérieux, ces dernières se dévoilent comme des espaces subversifs permettant de négocier l'identité culturelle officielle. Les festivités suivent ainsi un procédé de "folklorisation ", c'est-à-dire "de sélection des particularismes culturels les plus visuels des minorités pour faire leur "promotion" » (Grillot, 2001). Elles sont l'occasion, pour les $\mathrm{Na}$, d'une présentation de soi qui satisfait les projections touristiques auxquelles ils sont soumis, suivant une logique de mimesis (Adams, 1996), tout en permettant d'affirmer, dans l'interaction directe avec les touristes, leur contemporanéité et leur modernité. Les sociabilités sexuelles qui se jouent entre touristes et $\mathrm{Na}$, marquées par des projections romantiques ou hypersexualisées à l'égard des $\mathrm{Na}$, sont autant de ressources permettant de renégocier leurs positions sociales individuelles et collectives.

Dans cette même logique, le dernier chapitre compare les modalités des sociabilités sexuelles en contexte touristique et non touristique pour mettre en évidence les transformations contemporaines significatives affectant la reproduction sociale des maisonnées. Les divergences d'intérêt qui conduisent les $\mathrm{Na}$ et les touristes à entretenir des sociabilités sexuelles, les unes nourries par le désir de devenir moderne et/ou d'avoir une situation économique intéressante, les autres par des représentations hyper-sexu(alis)ées, montrent un changement des économies de l'intimité. Plutôt que d'être pensées au regard d'un ordre social collectif, et donc des impératifs et besoins pragmatiques de la maisonnée, les sociabilités sexuelles sont aujourd'hui pensées comme une opportunité pour transformer le statut social et économique individuel. La thèse démontre in fine que les nouvelles dynamiques sexuelles, qu'elles soient des relations avec les touristes, des imaginaires, des projections, etc., ont provoqué de nouveaux arrangements intimes. Cette forme d'individualisation recompose la société na et, en particulier, la configuration des maisonnées, avec pour conséquence une préférence pour la conjugalité, le mariage ou la cohabitation.

8 La thèse propose ainsi une description de la situation touristique, une notion offrant la possibilité de combiner la réflexivité ethnographique et l'analyse contextuelle afin de rendre compte de l'ambivalence des contextes locaux et de l'Histoire. Saisir le changement social à partir de cette grille de lecture permet de dépasser la problématique de l'impact ou de la capacité d'agir pour interroger les actions des différents acteurs du tourisme et de leurs intérêts et donner à voir les recompositions de l'organisation sociale. Au cœur de cette dernière, se trouvent les maisonnées (awo), une conception indigène des relations entre proches et des logiques de l'économie domestique, dont l'importance dans la vie sociale et la parenté permet de saisir avec finesse la flexibilité de la parenté na. 


\section{BIBLIOGRAPHIE}

Vincanne ADAMS, Tiger of the snow and other virtual Sherpas, Princeton University Press, 1996.

Georges BALANDIER, «La situation coloniale : approche théorique », les Classiques des sciences sociales, [1951] 2008 [http://classiques.uqac.ca/contemporains/balandier_georges/ situation_coloniale_1951/situation_coloniale_1951.html].

Hua CAI. Une société sans père ni mari : les Na de Chine, PUF, 1997.

Janet CARSTEN (dir.), Cultures of Relatedness: New Approaches to the Study of Kinship, Cambridge University Press, 2000.

Janet CARSTEN et Stephen HUGH-JONES, About the House: Lévi-Strauss and Beyond, Cambridge University Press, 1995.

Saskia CoUSIN et Bertrand RÉAU, Sociologie du tourisme, La Découverte, 2009.

Anne DOQUET, «L'enquête en "situation ethnologique" ou l'exercice nécessaire de la réflexivité », dans Olivier LESERVOISIER et Laurent VIDAL (dir.), L'anthropologie face à ses objets, Éditions des archives contemporaines, 2007.

Suzanne GILLESPIE, « Lévi-Strauss, maison and sociétés à maison », dans Rosemary JOYCE et Suzanne GILLEPSIE (dir.), Beyond Kinship, social and material reproduction in house societies, University of Pennsylvania Press, 2000.

Caroline GRILLOT, «L'impact de la folklorisation dans l'expression identitaire. La fête de Guzang chez les Miao de Xijiang », Ateliers, n² 24, 2001.

Stevan HARRELL, « Introduction: civilizing projects and the reaction to them », dans Stevan HARRELL (dir.), Cultural encounters on China's ethnic frontiers, University of Washington Press, 1995.

Elizabeth HSU, « Moso and Naxi House », dans Mickaël oPPITZ et Elizabeth HSU (dir.), Naxi and Moso Ethnography. Kin, Rites, Pictographs, Völkerkundemuseum, 1998.

Claude LÉVI-STRAUSS, La voie des masques, Plon, 1979.

Claude LÉVI-STRAUSS, Paroles données, Plon, 1984.

James SCOTT, La domination et les arts de la résistance : fragments du discours subalterne, Éditions Amsterdam, 2008.

Bertrand RÉAU et Franck POUPEAU, « L'enchantement du monde touristique », dans Saskia cousIN, Bertrand RÉAU et Yves WINKIN (dir.), Actes de la recherche en sciences sociales, vol. 170, n 5, 2007.

Yunxiang YAN, Private Life Under Socialism. Love, Intimacy, and Family in a Chinese Village, 1949-1999, Stanford University Press, 2003.

\section{NOTES}

1. Les termes chinois sont systématiquement indiqués en italique en utilisant d'abord le système de romanisation pinyin puis les sinogrammes. Les termes en langues na sont quant à eux indiqués en italique, mais ne sont pas suivis d'idéogrammes car il s'agit d'une langue orale ne disposant pas de système d'écriture. J'utilise un système de romanisation proche du français. 


\section{AUTEUR}

\section{PASCALE-MARIE MILAN}

Chercheure associée au LARHRA (UMR 5190) - Anthropologie

pascale-marie.milan[at]univ-lyon2.fr 